\title{
Inclusión social y laboral de las personas con discapacidad desde la articulación socio-sanitaria en el Perú
}

\author{
Luis Quiroz-Avilés', 3, 4 \\ Jorge Cordero-Valera', 2, 4 \\ Erika Giraldo-Vizcarra ${ }^{1,4}$
}

RESUMEN: La discapacidad es el resultado de la interacción de los problemas de salud, factores personales y factores ambientales. Por esta razón resulta ser muy variable, al igual que las desventajas que produce en la persona. En el Perú solo el 20\% de las personas con discapacidad tiene un empleo. Las brechas educativas, de acceso a atención de salud, prestaciones sociales y económicas y de inclusión social son parcialmente conocidas. Uno de los principales problemas de la población con discapacidad es que sigue siendo invisible. Afortunadamente, debido a políticas internacionales y nacionales, son cada vez más las personas con diferentes discapacidades que se hacen visibles y factibles de identificar y consultar respecto a sus necesidades. En Perú se realizan diversas acciones en materia de atención de las personas con discapacidad. Sin embargo, pocas carteras de servicios ofrecen una atención integral con resultados alentadores como la del seguro Social de Salud, que incluye prestaciones de salud, sociales y económicas.

PALABRAS CLAVE: Evaluación de la discapacidad; Servicios de salud; Apoyo social; Política de salud; Empleo.

Citar como: Quiroz L, Cordero J, Giraldo E. Inclusión social y laboral de las personas con discapacidad desde la articulación socio-sanitaria en el Perú. CASUS. 2017;2(3):179-184. 


\section{INTRODUCCIÓN}

A menudo cuando se habla de personas con discapacidad pensamos en una silla de ruedas o en una persona con síndrome de Down. Se suele creer que la discapacidad siempre es evidente y visible. La realidad es otra (1). Al interactuar los problemas de salud, factores personales y ambientales la discapacidad resulta ser muy variable, al igual que las desventajas que produce en una persona. Afortunadamente, debido a políticas internacionales y nacionales, son cada vez más las personas con diferentes discapacidades que se hacen visibles (2). Sin embargo, cuando gestionamos para su bienestar, no siempre tenemos en cuenta las necesidades de todas las personas con discapacidad. Esto podría dar como resultado que la formulación de políticas o estrategias no sea la mejor (2). En el mismo sentido, cuando consideramos a una persona con necesidad de atención sanitaria, sin tener en cuenta sus factores personales como edad, raza o sexo, así como los factores ambientales como el domicilio, el acceso al transporte, a lentes correctores o a un empleo, la intervención siempre será parcial e incompleta (3).

El Informe mundial sobre la discapacidad, publicado en el año 2011, hace referencia a datos de la Encuesta Mundial de Salud. El mismo indica que las personas con discapacidad tienen más del doble de probabilidades de considerar que los proveedores de asistencia de salud carecen de la competencia adecuada para atender sus necesidades (4). Igualmente señala una probabilidad cuatro veces mayor de ser tratadas mal, y una probabilidad tres veces mayor de que se les niegue la atención de salud necesaria (4). El Informe Mundial sobre personas con discapacidad menciona además que las personas con discapacidad son particularmente vulnerables a las deficiencias que presentan los servicios tales como la atención de salud, la rehabilitación y la asistencia y apoyo (4).

El entorno (incluido el lector) desempeña un papel crucial como facilitador para la participación plena de las personas con discapacidad en las actividades comunitarias, o como barrera, en caso de que la limite. En este sentido, cuando analizamos el mercado laboral para las personas con discapacidad, conceptos erróneos de los empleadores, por ejemplo, que las personas con discapacidad son menos productivas que sus homólogas sin discapacidad (5). Así como el desconocimiento de la implementación de los ajustes razonables establecidos en nuestro medio por la Ley
29973 - Ley General de la persona con Discapacidad vigente, o el incumplimiento de la cuota de empleo normada por el reglamento de la misma ley son elementos que limitan las oportunidades de empleo de este grupo vulnerable (6).

\section{PROBLEMÁTICA DE LA INCLUSIÓN SOCIAL Y LABORAL EN PERÚ}

A nivel nacional e internacional existen acuerdos y normas que respaldan el ejercicio de los derechos de las personas con discapacidad. Entre ellos se encuentran la Convención sobre los Derechos de las Personas con discapacidad, la Ley General de la Persona con Discapacidad (6), la Política Andina en salud para la prevención de la Discapacidad (7), el Plan de Igualdad de Oportunidades para las personas con discapacidad (8), Convenio $\mathrm{N}^{\circ} 159$ de la Organización Internacional del Trabajo (OIT) (9), así como servicios, programas y planes para la integración de las personas con discapacidad.

No obstante, las normas publicadas son escasas las acciones nacionales ejecutadas con resultados contundentes, que demuestren que nos encontramos en proceso de ser inclusivos; o que contamos con una cartera única de rehabilitación integral. Esta última entendida como la integración de la rehabilitación funcional, profesional y social (10).

De acuerdo a la segunda recomendación vertida en el Informe mundial sobre la Discapacidad; se debe invertir en programas y servicios específicos para las personas con discapacidad (4). En concordancia con dicho Informe el Seguro Social de Salud-EsSalud ha calculado que la inversión realizada en la rehabilitación profesional de una persona asegurada con discapacidad, a la que logran incluir en un puesto de trabajo es recuperada con los aportes de este mismo trabajador, como máximo en los dos primeros años de trabajo (9).

De acuerdo a cifras proporcionadas por la Gerencia de la Persona con Discapacidad de EsSalud son 1179 000 los asegurados que presentan discapacidad (10). Anualmente son más de 1600 las personas con discapacidad que logran incluirse laboralmente a través de sus servicios de rehabilitación profesional. La tendencia es al incremento de más de $30 \%$ anual en la cifra de colocaciones laborales en los tres últimos años (10).

Lo anterior se debe a que EsSalud ha fortalecido sus políticas de atención social y sanitaria de las personas que viven con algún tipo de discapacidad. También 
brinda una cartera de prestaciones sociales orientadas a la rehabilitación profesional y social. Esta se lleva a cabo en los Centros de Rehabilitación Profesional y social (CERPS) y los Módulos Básicos de Rehabilitación Profesional y Social (MBRPS), distribuidos en todo el país en número de 17 unidades operativas (10).

De acuerdo a la recomendación de diferentes fuentes, los servicios que generan inclusión social y laboral, brindados como prestaciones sociales deben ser considerados complementarios a las prestaciones de salud y económicas brindadas por otros sectores, o por la misma institución, como es el caso de EsSalud en el Perú (7).

De acuerdo a cifras obtenidas en la primera Encuesta Especializada sobre Discapacidad realizada por el INEI en el año 2012 el $58.2 \%$ de las personas con discapacidad ocupadas de 14 y más años son trabajadores independientes (11). En contraste, en EsSalud solo el $40 \%$ de las personas con discapacidad son colocadas en trabajos independientes pues resulta mayor la contratación como empleados u obreros (10, 11).

El Convenio $\mathrm{N}^{\circ} 159$ de la OIT sobre la readaptación profesional y el empleo de las personas con discapacidad, señala que es un deber estatal promover programas de mantenimiento del empleo dirigidos a estas personas (9). Sin embargo, lograr que una empresa o institución pública contrate una persona con discapacidad no es tarea sencilla. Más difícil aún puede resultar que esa persona sea empleable. Es decir, que cuente con un perfil laboral atractivo para el potencial empleador; o que el puesto de trabajo sea el adecuado: sin barreras para esa persona. En la actualidad, a pesar de la cuota de empleo establecida por la Ley 29973, sin intervención la inclusión laboral de una persona con discapacidad puede no darse nunca (6). Esto en gran parte debido al desconocimiento de la empresa de las acciones a desarrollar para tal efecto, como por ejemplo la implementación de ajustes razonables.

La OIT estima que el $80 \%$ de las personas con discapacidad en edad de trabajar están en situación de desempleo, lo que se debería principalmente a la falta de oportunidades educativas y de capacitación laboral, así como al prejuicio entre los empleadores de que es una población incapacitada para el trabajo (7). En ese sentido, el Ministerio de Trabajo y Promoción del Empleo, en los últimos años viene aprobando documentos técnicos, e implementando sus servicios dirigidos a mejorar el acceso de las personas con discapacidad a un trabajo formal (12).

Por otro lado, la mejora de la calidad de vida de las personas con discapacidad resulta un objetivo reiterado en diferentes programas y carteras dirigidas a este grupo vulnerable. Sin embargo, si buscamos la atención integral a personas con discapacidad con una mirada socio sanitaria, concierne también pensar en los cuidados paliativos para las personas con discapacidad. Este componente social se encuentra en desarrollo incipiente en EsSalud y no forma parte aún de la oferta de alguna institución de forma estructurada (10).

Otro punto relevante en esta situación es el proceso de capacitación realizado por el Ministerio de salud del Perú a médicos certificadores de discapacidad en coordinación con EsSalud y las FFAA. Con la finalidad de acortar la brecha de Certificados de Discapacidad emitidos (13). Este documento acredita a la persona con discapacidad. Para el 2015 se identificó que solo el $6 \%$ de las personas con discapacidad contaban con este certificado (11). No contar con este documento conduce a la restricción en la participación de la persona. Puesto que se convierte en uno de los requisitos para acceder a la pensión no contributiva para personas con discapacidad severa y en condición de pobreza extrema brindada por el Estado. Pese a los esfuerzos, el número de médicos certificadores resulta insuficiente aun para atender la demanda de al menos el 5.2\% de la población peruana, estimada por la Encuesta Especializada de Discapacidad ENEDIS-2012, como la población con discapacidad en el Perú (11).

Otro aspecto importante es la intervención de la familia como principal soporte de las personas con discapacidad. Los programas dirigidos a capacitarla y hacerla parte del proceso de integración e inclusión social y laboral se desarrollan con mayor auge en los últimos años $(7,8)$. Así, EsSalud cuenta con un Programa de Escuela de Familias y Discapacidad, con el objetivo de empoderar a la familia como parte del proceso de integración e inclusión de las personas con discapacidad, el mismo que da origen al componente social de los cuidados paliativos (10).

Mención aparte merece el factor educación. Según cifras del INEI, la población con discapacidad cuenta con marcadas desventajas para acceder a educación, formación y por ende empleo (11). Este tema debiera ser abordado en profundidad por el sector 
correspondiente. De forma general, podemos decir que, según cifras publicadas por el INEI, el $24 \%$ de las personas con discapacidad del Perú, no cuenta con ningún nivel educativo. El $41 \%$ tiene solo primaria, el $23 \%$ secundaria y solo el $12 \%$ ha realizado estudios superiores (11). Estas cifras evidencian la urgencia de mejorar la calificación laboral, a fin de favorecer su inclusión en el mercado de trabajo (5).

\section{CONSIDERACIONES FINALES}

Inclusión significa que las leyes, políticas, planes y servicios de la comunidad deben adaptarse, planificarse y organizarse para garantizar el libre, pleno e independiente desarrollo de las personas, basado en el respeto y aceptación de sus diferencias, capacidades y necesidades $(5,9)$. Aunque son muchos los sectores nacionales, las organizaciones de personas con discapacidad y sus familias, empresas privadas y otros que han empezado a adoptar medidas para mejorar la vida de las personas con discapacidad (5-9), es mucho lo que queda por hacer. Esto en parte se debe a que la discapacidad es un fenómeno complejo y puede ser difícil de medir y de identificar.

El Informe mundial sobre la discapacidad enfatiza que: la rehabilitación es una buena inversión porque genera capacidad humana (...) habría que centrar la atención en mejorar la eficiencia y eficacia, ampliando la cobertura y mejorando la calidad y asequibilidad (4). Al analizar el costo-beneficio del servicio de rehabilitación profesional, cuyo resultado es la contratación de personas con discapacidad, se ha identificado que la inversión realizada se recupera en dos años, a través de los correspondientes aportes, luego de su contratación (4). Crear entornos favorables eliminando los obstáculos que limitan el acceso al transporte, información y comunicación hará posible que las personas con discapacidad participen en la educación, empleo y vida social, reduciendo así su aislamiento y dependencia.

Los resultados positivos que tiene EsSalud en la rehabilitación profesional y social, se basan en la articulación socio-sanitaria (10). Es decir, no basta la rehabilitación física per se, si no es integrada a la reinserción social y laboral de la persona. Para ello es fundamental la participación de la familia con el inicio del componente social de los cuidados paliativos. Este incluye un trato digno, afecto, mejora de la autoestima, no discriminación y fortalecimiento de la autonomía e independencia de la persona que vive con discapacidad.

\section{RECOMENDACIONES}

El fortalecer el acceso a la educación y formación de las personas con discapacidad, así como el desarrollar las competencias del docente en materia de discapacidad, será un importante paso para mejorar las condiciones laborales de las personas con discapacidad, pues el bajo nivel educativo constituye la principal desventaja en este grupo vulnerable.

Por otro lado, la sensibilización y concientización de los empleadores respecto a las potencialidades de las personas con discapacidad constituyen estrategias fundamentales para lograr la integración laboral de las personas en edad laboral. Asimismo, el articular los servicios, entre ellos los socio-sanitarios, dirigidos a este grupo, logrará de forma integral y eficiente la integración e inclusión social y laboral de las personas con discapacidad y su familia, siendo esta última en apoyo fundamental en este proceso.

Finalmente, resultan aún insuficientes las carteras de servicios con un enfoque centrado en la persona y con nivel aceptable de articulación socio sanitaria, siendo la rehabilitación un proceso que ineludiblemente debe cumplir con estas características. EsSalud y sus positivos resultados en atención integral a las personas con discapacidad brinda cobertura al 30\% de la población nacional general. Por ello, es urgente incrementar la oferta a nivel nacional, de modo que sea accesible a todos.

\section{REFERENCIAS BIBLIOGRÁFICAS}

1. Climent JM, Rodríguez C, Mondéjar F. Evolución histórica de la asistencia de rehabilitación: desde un modelo hospitalario a un modelo descentralizado. Rehabilitación. 2011;45 (1):2-7.

2. Cotanda FL. Una perspectiva de la política sanitaria 20 años después del Informe Lalonde. Gaceta Sanitaria. 1994;8(43):189194.

3. López O, Canga N, Mujika A, Pardavila MI, Belintxon M, Serrano I, et al. Cinco paradojas de la promoción de la salud. Gaceta Sanitaria. 2017; 31(3):269-272.

4. Organización Mundial de la Salud-OMS. El Informe Mundial sobre personas con discapacidad [internet]. Ginebra, Suiza: OMS; 2011 [citado el 1 de noviembre 2017]. Disponible en: http://www.who.int/disabilities/world_report/2 011/summary_es.pdf 
5. Asociación Pro Derechos HumanosAPRODEH. Guía para la inclusión laboral de personas con discapacidad intelectual, psicosocial y autismo [internet]. Lima, Perú: APRODEH; 2017 [citado el 1 de noviembre de 2017]. Disponible en: http://www.aprodeh.org.pe/documentos/publi caciones/discapacidad/Guia_para_la_Inclusio n_Laboral_de_Personas_con_discapacidad_in telectual_psicosocial_y_autismo.pdf

6. Ministerio de la mujer y poblaciones vulnerables- MIMP. Ley 29973 - Ley General de la persona con Discapacidad [internet]. Lima, Perú: MINP; 2012 [citado el 1 de noviembre de 2017]. Disponible en: https://www.mimp.gob.pe/webs/mimp/herra mientas-recursos-violencia/contenedordgcvg-recursos/contenidos/Legislacion/Leygeneral-de-la-Persona-con-Discapacidad29973.pdf

7. Organismo Andino de Salud-Convenio Hipólito Unanue ORAS-CONHU. Política andina en salud para la prevención de la discapacidad y para la atención, habilitación/ Rehabilitación integral de las personas con discapacidad [internet]. Lima, Perú: ORAS-CONHU; 2010 [citado el 2 de noviembre de 2017]. Disponible en: http://www.orasconhu.org/sites/default/files/L ibro\%20Politica\%20Andina\%20de\%20Disca pacidad.pdf

8. Ministerio de la Mujer y Poblaciones Vulnerables-MIMP. Plan de igualdad de oportunidades para personas con discapacidad 2016-2021 [internet]. Lima, Perú: MIMP [citado el 3 de noviembre de 2017]. Disponible en: https://www.mimp.gob.pe/files/planes/planigualdad-de-Oportunidades.pdf

9. Organización Internacional del Trabajo (OIT). Convenio $\quad \mathrm{N}^{\circ}$ 159 sobre la readaptación profesional y empleo de personas inválidas. Ginebra, Suiza: 1983 [citado el 2 de noviembre de 2017]. Disponible en: http://www.ilo.org/dyn/normlex/es/f?p=NOR MLEXPUB:12100:0::NO::P12100_INSTRU MENT_ID:312304

10. Estudio Bio-Socio-Demográfico y Análisis de la Oferta
Demanda de la población con Discapacidad asegurada por ESSALUD. 2017

11. Instituto Nacional de Estadísticas e Informática. Primera encuesta nacional especializada sobre discapacidad [internet]. Lima, Perú: INEI; 2012 [citado el 1 de noviembre de 2017]. Disponible en: ://www.inei.gob.pe/media/MenuRecursivo/pu blicaciones_digitales/Est/Lib1171/ENEDIS\%2 02012\%20-\% 20COMPLETO.pdf

12. Ministerio de Trabajo y promoción de la salud-MTP. Personas con discapacidad tendrán empleos adecuados e igualdad de condiciones [internet]. Lima, Perú: MTP; 2016 [citado el 2 de noviembre de 2017]. Disponible en: http://www2.trabajo.gob.pe/prensa/notas-deprensa/personas-con-discapacidad-tendranempleos-adecuados-e-igualdad-decondiciones-laborales/

13. Ministerio de salud-MINSA. Norma técnica para evaluación, calificación y certificación de la persona con discapacidad [internet]. Lima, Perú: MINSA; 2016 [citado el 3 de noviembre]. Disponible en: $\mathrm{ftp} / / \mathrm{ftp} 2 . m i n s a . g o b . p e /$ normaslegales/2016/R M-_N_981-2016-MINSA.pdf 


\section{Social and occupational inclusion of people with disabilities from the socio-health articulation in Peru}

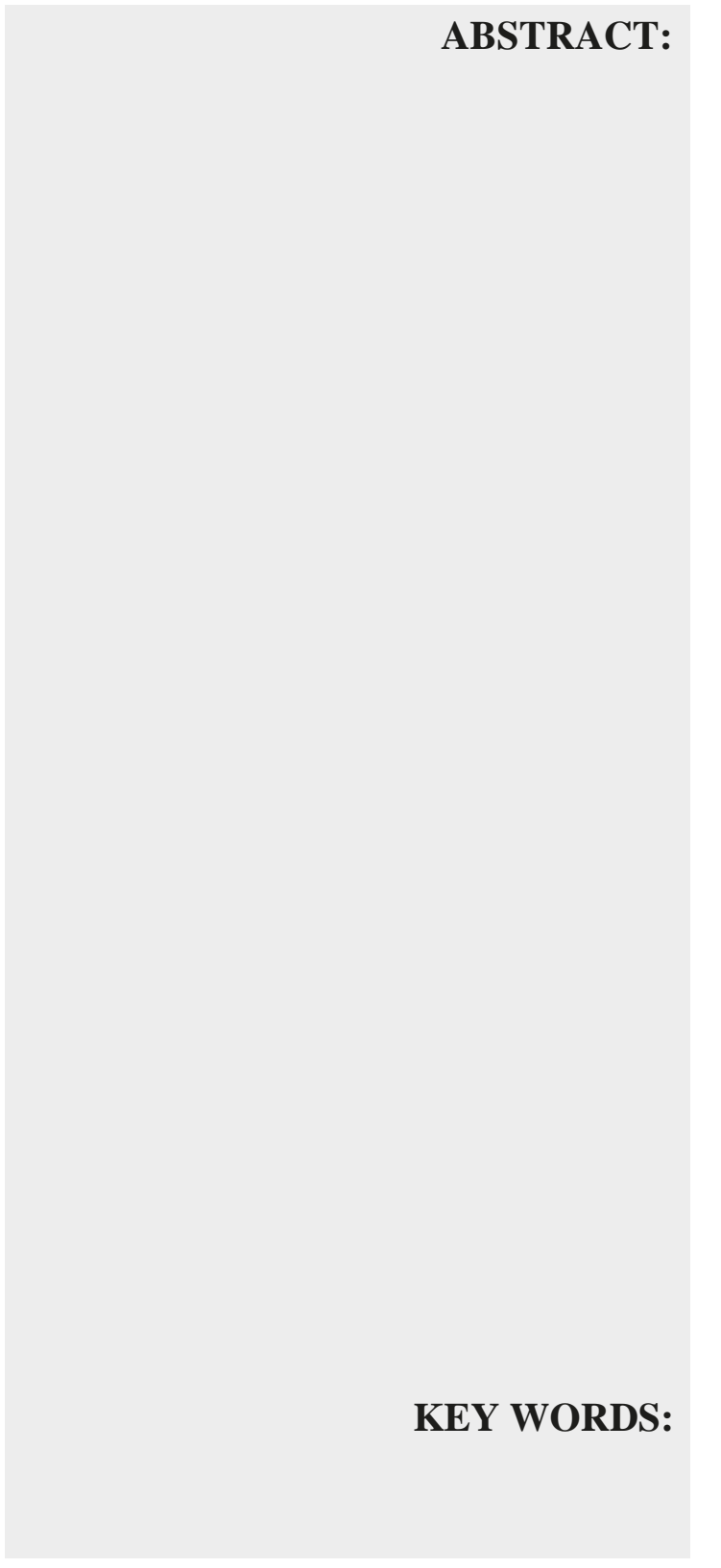

Disability is the result of the interaction of health problems, personal factors, and environmental factors. For this reason, it turns out to be very variable, as well as the disadvantages it produces in the person. In Peru, only $20 \%$ of people with disabilities have a job. The educational gaps, access to health care, social and economic benefits and social inclusion are partially known. One of the main problems of the population with disabilities is that it is still invisible. Fortunately, due to international and national policies, more and more people with different disabilities are becoming visible and feasible to identify and consult with respect to their needs. In Peru, various actions are carried out regarding the care of people with disabilities. However, few portfolios of services offer comprehensive care with encouraging results, such as the Social Health Insurance, which includes health, social and economic benefits.

Disability evaluation; Health services; Social support; Health policy; Employment. 